Research in Astronomy and Astrophysics manuscript no.

(LTEX: raa.tex; printed on November 8, 2018; 3:40)

\title{
Early afterglows from radially structured outflows and the application to X-ray shallow decays
}

\author{
Xue-Wen Liu ${ }^{\star 1,2,3}$, Xue-Feng Wu ${ }^{\star \star 1,3,4}$, Yuan-Chuan Zou ${ }^{\star \star \star 5}$ and Tan $\mathrm{Lu}^{\dagger 1,3}$ \\ ${ }^{1}$ Purple Mountain Observatory, Chinese Academy of Sciences, Nanjing, 210008, China \\ ${ }^{2}$ Graduate School, Chinese Academy of Sciences, Beijing, 100039, China \\ ${ }^{3}$ Joint Center for Particle Nuclear Physics and Cosmology (J-CPNPC), Nanjing 210093, China \\ ${ }^{4}$ Department of Astronomy \& Astrophysics, Pennsylvania State University, University Park, PA 16802, \\ USA \\ ${ }^{5}$ Department of Physics, Huazhong University of Science and Technology, Wuhan 430074, China
}

\begin{abstract}
In the fireball model, it is more physically realistic that gamma-ray burst (GRB) ejecta have a range of bulk Lorentz factors (assuming $M \propto \Gamma^{-s}$ ). The low Lorentz factor part of the ejecta will catch up with the high Lorentz factor part when the latter is decelerated by the surrounding medium to a comparable Lorentz factor. Such a process will develop a long-lasting weak reverse shock until the whole ejecta are shocked. Meanwhile, the forward shocked materials are gradually supplied with energy from the ejecta that are catching-up, and thus the temporal decay of the forward shock emission will be slower than that without an energy supply. However, the reverse shock may be strong. Here, we extend the standard reverse-forward shock model to the case of radially nonuniform ejecta. We show that this process can be classified into two cases: the thick shell case and the thin shell case. In the thin shell case, the reverse shock is weak and the temporal scaling law of the afterglow is the same as that in Sari \& Mészáros (2000). However, in the thick shell case, the reverse shock is strong and thus its emission dominates the afterglow in the high energy band. Our results also show slower decaying behavior of the afterglow due to the energy supply by low Lorentz factor materials, which may help the understanding of the plateau observed in the early optical and $\mathrm{X}$-ray afterglows.
\end{abstract}

Key words: gamma-rays: bursts - hydrodynamics - radiation mechanisms: nonthermal shock waves

\section{INTRODUCTION}

The central engine and surrounding environment provide the most important insights to the mystery of gamma-ray bursts (GRBs), the most violent explosions in the universe. Thanks to BeppoS $A X$, owing to its 
ability to accurately locate objects, the first afterglow of a GRB was discovered in 1997 (Costa et al. 1997). Afterward, broad band data of afterglows were achieved, and were fitted using the standard fireball-shock model (Rees \& Mészáros 1992, 1994; Mészáros \& Rees 1993, 1997; for reviews, see Zhang 2007). The parameters of GRBs, such as the total burst energy, the type (ISM or wind) and number density of the environment, and the electron and magnetic field equipartition factors were then constrained (e.g., Wu et al. 2003; Fan et al. 2002; Zhang et al. 2003). Early afterglows can even be used to constrain the initial Lorentz factors of GRB fireballs (Molinari et al. 2007; Xue et al. 2009). It is believed that early afterglows are produced by reverse-forward shocks when relativistic ejecta interact with the circum-burst medium, which was first studied by Rees \& Mészáros (1992) and Sari \& Piran (1995). Then GRB 990123, a remarkable event with a bright early optical flash was discovered (Akerlof et al. 1999), which was interpreted by the reverse-forward external shock model well(Sari \& Piran 1999). Much attention was consequently focused on early afterglow radiation, considering the effects of circum-burst environments and non-relativistic reverse shocks (Kobayashi 2000; Wu et al. 2003; Zou et al. 2005). Subsequently, more optical flashes were observed in, e.g., GRBs 021211, 050525a, 060111B, 060117B and 080319B (Wei 2003; Shao \& Dai 2005; Klotz et al. 2006; Jelinek et al. 2006; Racusin et al. 2008). These works were based on the assumption that the Lorentz factor does not change in the shell. The whole light curve from the reverse-forward external shock has two types: re-brightening (Type I) and flattening (Type II) (Zhang et al. 2003). In some GRBs, such as GRBs 050319, 060206, 060210 and 060313, the early optical light curves have a plateau which is difficult explain within the uniform ejecta model (UEM). Meanwhile, the shallow decay of the canonical X-ray afterglow behavior discovered in the Swift era (Nousek 2006; Zhang et al. 2006; O'Brien et al. 2006) remains a matter of debate.

Because of the above problems, we reconsider the baryon-dominated energy injection model in which the ejecta have a wide $\Gamma$-distribution: the part of the ejecta with low Lorentz factor lagging behind the one with high Lorentz factor. The low- $\Gamma$ part catches up with the high- $\Gamma$ part when the latter is decelerated to a comparable Lorentz factor, so the reverse shock is usually mildly relativistic and mainly contributes to the far-IR or millimeter band (Rees \& Mészáros 1998; Sari Mészáros 2000). Once the reverse shock starts, it will travel through the whole ejecta from the front highest- $\Gamma$ part to the rear lowest- $\Gamma$ part. Based on the treatment widely adopted in the UEM, we reconsider this issue by assuming a given distribution of Lorentz factors in the ejecta. We calculate the dynamic evolution of the reverse-forward shocks produced by this radially structured ejecta propagating into the circum-burst medium, and present the analytical and numerical results.

We organize our paper as follows. In $\S 2$, we describe the dynamics of the reverse-forward shocks including the thick shell case and the thin shell case, respectively. We discuss the reverse shock emission in $\S 3$. The numerical results are shown in $\S 4$. Finally, we present a brief discussion in $\S 5$.

\section{DYNAMICS OF THE EJECTA WITH A $\Gamma$-DISTRIBUTION}

As Rees \& Mészáros (1998) postulated, the central engine of GRBs may eject relativistic shell-like ejecta with a range of Lorentz factors 
Such ejecta have an energy $\Gamma M c^{2} \propto \Gamma^{-s+1}$. Applying the model to observations shows that the value of the index $s$ is typically $\sim 2.5$ (Zhang et al. 2006; Nousek et al. 2006), which is larger than the suggested value $\sim 1.5$ (e.g., Rees \& Mészáros 1992), so the low- $\Gamma$ mass carries more kinetic energy than predicted. When the ejecta interact with the circum-burst medium, a pair of shocks emerges: a forward shock propagating into the circum-burst medium and a reverse shock propagating into the shell. There are four regions separated by the two shocks: (1) the unshocked circum-burst medium, (2) the shocked medium, (3) the shocked shell material and (4) the unshocked shell material. Using the shock jump condition and the equality of pressure and velocity along the contact discontinuity, the Lorentz factor $\Gamma$ and the number density in both shocked media can be determined by the density of the circum-burst medium $n_{1}$ and the unshocked shell $n_{4}$ (Blandford \& McKee 1976, hereafter BM). Here, the number density of the unshocked shell is nonuniform, depending on the Lorentz factor distribution in the shell

$$
n_{4}=\frac{|(d M(>\Gamma) / d \Gamma)(d \Gamma / d x)|}{4 \pi r^{2} m_{p} \Gamma(x)}
$$

We assume an initial Lorentz factor distribution in the shell

$$
\Gamma \sim \Gamma_{\min }\left[\frac{x(t=0, \Gamma)}{\Delta_{0}}\right]^{-1 / b}
$$

where $\Delta_{0}$ and $\Gamma_{\min }$ are the initial width and minimum Lorentz factor of the shell, $x(t=0, \Gamma)$ represents the initial position in the shell with the origin located at the outer edge of the shell (See Fig.11). Due to the distribution of Lorentz factors, the shell will spread with time, then the value of $x$ of a fixed element will become larger and larger,

$$
x(t, \Gamma)=x(t=0, \Gamma)+\left(\beta_{\max }-\beta\right) c t,
$$

where $\beta=\sqrt{1-1 / \Gamma^{2}}$. Thus the Lorentz factor distribution in the shell at any time is determined.

The properties of the shocks are largely determined by the parameter defined as

$$
f \equiv \frac{n_{4}}{n_{1}}=\frac{s M_{b} \Gamma_{\min }^{s} \Gamma^{-s-2}}{4 \pi r^{2} m_{p} n_{1}}\left|\frac{d \Gamma}{d x}\right|
$$

where $M_{b}$ is the total mass of the shell. Eq. (1) can be written as $M(>\Gamma) \approx M_{b} \Gamma_{\min }^{s} \Gamma^{-s}$ (here $\Gamma_{\min } \ll$ $\Gamma_{\max }$ is assumed). Combining Eq. (4) and (5), we need another equation to describe the evolution of the radius and Lorentz factor of the shocks: the relation between the distance $d x$ which the reverse shock travels in the shell and the distance $d r$ which the shell propagates in the circum-burst medium in the same time interval is (Kobayashi 2000)

$$
d r=\alpha \Gamma f^{1 / 2} d x
$$

where $r$ is the radius of the shell and the parameter $\alpha$ is $\sim 1$.

There are two approximations under which the shock evolution can be described analytically: the thick shell case and the thin shell case, depending on the significance of the spreading effect in the last term in Eq. (4). If the spreading term is larger than the initial $x(t=0, \Gamma)$, the shell is regarded as a thin shell, otherwise it is a thick shell. Below, we will consider these two cases separately.

\subsection{Thick Shell Case}

In the thick shell case, the spreading effect can be ignored, so the width of the shell always remains at its 
We can get from Eqs. (3) and (4)

$$
d x=-b \Delta_{0}\left(\frac{\Gamma}{\Gamma_{\min }}\right)^{-b} \frac{d \Gamma}{\Gamma} .
$$

We can now calculate the comoving number density $n_{4}$ and the density ratio $f$. According to Eq. (5), we have

$$
\frac{d r}{d x}=\frac{\alpha^{2} s M_{b} \Gamma_{\min }^{s} \Gamma^{-s}}{4 \pi r^{2} m_{p} n_{1}}\left|\frac{d \Gamma}{d r}\right|
$$

In general, the number density of the circum-burst medium can be modeled as $n_{1}=A r^{-k}$. Specifically, $A=n_{1}=1.0 n_{1,0} \mathrm{~cm}^{-3}$ for an ISM environment $(k=0)$ and $A=3 \times 10^{35} A_{*} \mathrm{~cm}^{-1}$ for a free wind environment $(k=2)$ (Chevalier $\&$ Li 2000). Throughout this work, we adopt the convention $Q_{x}=Q / 10^{x}$ in cgs units. The solution of Eq. (8) reads

$$
r^{4-k}=\left(\frac{4-k}{s+b-1}\right)^{2} \frac{b(s-1) E \Delta_{0} \alpha^{2}}{4 \pi A m_{p} c^{2}}\left(\frac{\Gamma}{\Gamma_{\min }}\right)^{-(s+b-1)} .
$$

Keep in mind that $d r=2 \Gamma^{2} c d t$, the evolution of the Lorentz factor and radius of the ejecta with time can now be described. Just when the reverse shock crosses the shell, the Lorentz factor is equal to $\Gamma_{\min }$ and the shell reaches the crossing radius

$$
\begin{aligned}
r_{\Delta} & =\left[\left(\frac{4-k}{s+b-1}\right)^{2} \frac{b(s-1) E \Delta_{0} \alpha^{2}}{4 \pi A m_{p} c^{2}}\right]^{\frac{1}{4-k}} \\
& =\left\{\begin{array}{ll}
9.4 \times 10^{16} \alpha^{1 / 2}\left[\frac{b(s-1)}{(s+b-1)^{2}}\right]^{1 / 4} E_{53}^{1 / 4} \Delta_{0,12}^{1 / 4} n_{1,0}^{-1 / 4} \mathrm{~cm}, & (k=0) \\
8.5 \times 10^{15} \alpha\left[\frac{b(s-1)}{(s+b-1)^{2}}\right]^{1 / 2} E_{53}^{1 / 2} \Delta_{0,12}^{1 / 2} A_{*}^{-1 / 2} \mathrm{~cm}, & (k=2)
\end{array} .\right.
\end{aligned}
$$

In the thick shell case, the spreading effect is always negligible which requires $r_{\Delta} \leq \Gamma_{\min }^{2} \Delta_{0}$. Thus a lower limit of the minimal Lorentz factor must be satisfied

$$
\Gamma_{\min } \geq\left\{\begin{array}{l}
310\left[\frac{b(s-1)}{(s+b-1)^{2}}\right]^{1 / 8} \alpha^{1 / 4} E_{53}^{1 / 8} \Delta_{0,12}^{-3 / 8} n_{1,0}^{-1 / 8}, \quad(k=0) \\
92\left[\frac{b(s-1)}{(s+b-1)^{2}}\right]^{1 / 4} \alpha^{1 / 2} E_{53}^{1 / 4} \Delta_{0,12}^{-1 / 4} A_{*}^{-1 / 4}, \quad(k=2)
\end{array} .\right.
$$

Whether or not the reverse shock is relativistic depends on the parameter

$$
\left.\frac{f}{\Gamma^{2}}\right|_{\Gamma_{\min }} \approx\left\{\begin{array}{ll}
5.75 \frac{(s+b-1) \sqrt{s-1}}{b^{3 / 2}} \alpha^{-1} E_{53}^{1 / 2} n_{1,0}^{-1 / 2} \Delta_{0,12}^{-3 / 2} \Gamma_{\min , 2}^{-4}, & (k=0) \\
0.17 \frac{s-1}{b} E_{53} A_{*}^{-1} \Delta_{0,12}^{-1} \Gamma_{\min , 2}^{-4}, & (k=2)
\end{array} .\right.
$$

It can be seen that the parameter $f / \Gamma^{2}$ is smaller than one for the typical wind case $(\mathrm{k}=2)$ and for a dense ISM case $\left(n_{1}=100\right)$ at the crossing time, and before the crossing time it is proportional to $\Gamma^{2(3 b-b k+2 k-s-7) /(4-k)}$. If the Lorentz factor distribution is not too steep $0<b<3$ and the mass distribution index has a typical value of $1.5 \leq s \leq 2.5, f<\Gamma^{2}$ holds for the entire reverse-forward shocks interaction period which means that the reverse shock is relativistic all along. While in the other parameter space, it is possible that $f \gg \Gamma^{2}$ at the initial stage when the reverse shock is non-relativistic, and then $f \leq \Gamma^{2}$ which corresponds to the reverse shock evolving from being non-relativistic to being relativistic. If the reverse shock is relativistic, the relative Lorentz factor $\gamma_{34}$ between the shocked shell and the unshocked 


\subsection{Thin Shell Case}

In the thin shell case, the spreading effect is dominant. The position of an element in the shell, $x$, can be approximated by

$$
x(\Gamma) \approx\left(\beta_{\max }-\beta\right) c t \approx \frac{r}{2 \Gamma^{2}} .
$$

the parameter $f$ can be written as

$$
f=\frac{(s-1) E}{4 \pi r^{3} m_{p} c^{2} n_{1}}\left(\frac{\Gamma}{\Gamma_{\min }}\right)^{-(s-1)} .
$$

Based on the same procedure applied in the thick shell case, we can obtain the relation between the radius and the Lorentz factor

$$
r^{3-k}=\left(\frac{3-k}{s+1}\right)^{2} \frac{\alpha^{2}(s-1) E}{4 \pi A m_{p} c^{2} \Gamma_{\min }^{2}}\left(\frac{\Gamma}{\Gamma_{\min }}\right)^{-(s+1)} .
$$

Then the crossing radius is

$$
\begin{aligned}
r_{\Delta} & =\left[\left(\frac{3-k}{s+1}\right)^{2} \frac{\alpha^{2}(s-1) E}{4 \pi A m_{p} c^{2} \Gamma_{\min }^{2}}\right]^{\frac{1}{3-k}} \\
& = \begin{cases}1.7 \times 10^{17}\left[\frac{\alpha^{2}(s-1)}{(s+1)^{2}}\right]^{1 / 3} E_{53}^{1 / 3} n_{1,0}^{-1 / 3} \Gamma_{\min , 2}^{-2 / 3} \mathrm{~cm}, & (k=0) \\
1.8 \times 10^{15}\left[\frac{\alpha^{2}(s-1)}{(s+1)^{2}}\right] E_{53} A_{*}^{-1} \Gamma_{\min , 2}^{-2} \mathrm{~cm}, & (k=2)\end{cases}
\end{aligned}
$$

In contrast to the thick shell case, an upper limit of the minimal Lorentz factor must be satisfied to keep the thin shell assumption valid all along

$$
\Gamma_{\min } \leq\left\{\begin{array}{ll}
290\left[\frac{\alpha^{2}(s-1)}{(s+1)^{2}}\right]^{1 / 8} E_{53}^{1 / 8} \Delta_{0,12}^{-3 / 8} n_{1,0}^{-1 / 8}, & (k=0) \\
65\left[\frac{\alpha^{2}(s-1)}{(s+1)^{2}}\right]^{1 / 4} E_{53}^{1 / 4} \Delta_{0,12}^{-1 / 4} A_{*}^{-1 / 4}, & (k=2)
\end{array} .\right.
$$

For the thin shell case, it is interesting that $f / \Gamma^{2} \equiv \alpha^{-2}(s+1 / 3-k)^{2}$, which means that the reverse shock is always mildly relativistic $\left(\gamma_{34}-1 \approx \Gamma^{2} / f \sim 1\right)$.

\section{REVERSE SHOCK EMISSION}

Now that the dynamic related parameters, i.e., $\Gamma, \gamma_{34}$ and $n_{4}$ are determined, the radiation related properties of the shocked materials, such as the strength of the magnetic field $B_{i}^{\prime}$, the minimum Lorentz factor $\gamma_{m, i}^{\prime}$, the cooling Lorentz factor $\gamma_{c, i}^{\prime}$ and the number $N_{e, i}$ of shocked electrons can be determined. For the shocked region, the fraction $\epsilon_{B}$ and $\epsilon_{e}$ of the internal energy are assumed to be carried by magnetic fields and shockaccelerated electrons, respectively. The co-moving magnetic field is equal to $\sqrt{8 \pi \epsilon_{B} e_{i}^{\prime}}$, where the internal energy density $e_{i}^{\prime}=\left(\gamma_{\mathrm{rel}}-1\right) m_{p} c^{2} n_{i}$ (for the forward shock $\gamma_{\mathrm{rel}} \equiv \gamma_{2} \approx 1 / 2 \Gamma^{1 / 2} f^{1 / 4}$; for the reverse shock $\left.\gamma_{\mathrm{rel}} \equiv \gamma_{34}\right)$. The minimum Lorentz factor $\gamma_{m, i}^{\prime}=\epsilon_{e} C_{p}\left(m_{p} / m_{e}\right)\left(\gamma_{\mathrm{rel}}-1\right)$ with $C_{p} \equiv(p-2) /(p-1)$, the cooling Lorentz factor $\gamma_{c, i}^{\prime}=6 \pi m_{e} c /\left(\sigma_{T} B_{i}^{\prime 2} \gamma_{i} t\right)$. The increase of the number of shocked electrons is $d N_{e, 3}=4 \pi r^{2} \Gamma n_{4} d x$ for the reverse shocked region and $d N_{e, 2}=4 \pi r^{2} n_{1} d r$ for the forward shocked region. In the standard synchrotron radiation model, the two characteristic frequencies and the peak flux density are

$$
\nu_{m, i}=\frac{q_{e} B_{i}^{\prime}}{2 \pi m_{e} c} \gamma_{m, i}^{\prime 2} \gamma_{i}, \quad \nu_{c, i}=\frac{q_{e} B_{i}^{\prime}}{2 \pi m_{e} c} \gamma_{c, i}^{\prime 2} \gamma_{i}, \quad F_{\nu, \max , \mathrm{i}}=\frac{N_{e, i}}{4 \pi d_{L}^{2}} \frac{m_{e} c^{2} \sigma_{T}}{3 q_{e}} B_{i}^{\prime} \gamma_{i},
$$

where $d_{L}$ is the luminosity distance of a GRB, $q_{e}$ is the charge of electron and $\sigma_{T}$ is the Thomson cross 
listed in Table 1 for both the forward shock and reverse shock, and for both the thick shell case and thin shell case.

The distinct discrepancy between the nonuniform ejecta model (NUEM) and the UEM is the reverse shock emission. Once the reverse shock has crossed the shell, the forward shock and shocked region begin to approach the Blandford-McKee (BM) solution (Kobayashi et al. 1999), and the following light curve is the same as that in the UEM. Below we only discuss the synchrotron emission from the reverse-shocked region before the crossing time. Since we have already obtained the temporal indices of $\nu_{m}, \nu_{c}$ and $F_{\max }$, in the following we only need to know the values of the characteristic frequencies and the peak flux density at the crossing time so we can extrapolate the early light curve back in time from the reverse shock.

\subsection{Thick Shell Case}

The reverse shock in the thick shell case we consider here is assumed to be relativistic $\left(\gamma_{34}-1 \approx \gamma_{34}\right)$. The crossing time $T_{\Delta}$ is $\sim \Delta / c$, when the Lorentz factor of the shell is $\Gamma_{\min }$ and the number of the shocked electrons is the total number of electrons in the ejecta, i.e., $N_{e, 3}=E(s-1) /\left(s m_{p} c^{2} \Gamma_{\min }\right)$. According to Eq. (18), we have

$$
\begin{gathered}
\nu_{m} \sim 4.0 \times 10^{13}(1+z)^{-1}\left(\frac{p-2}{p-1}\right)^{2} \epsilon_{e,-0.5}^{2} \epsilon_{B,-2}^{1 / 2} n_{1,0}^{1 / 2} \Gamma_{\min , 2.5}^{2} \mathrm{~Hz}, \\
\nu_{c} \sim 1.0 \times 10^{17}(1+z)^{-1 / 2} \sqrt{\frac{\alpha^{2} b^{3}}{(s+b-1)^{2}(s-1)}} \epsilon_{B,-2}^{-3 / 2} E_{53}^{-1 / 2} n_{1,0}^{-1} T_{\Delta,-2}^{-1 / 2} \mathrm{~Hz}, \\
F_{\nu, \max } \sim 1.36(1+z)^{7 / 4}\left[\frac{(s-1)^{5}(s+b-1)^{2}}{\alpha^{2} b^{3} s^{4}}\right]^{1 / 4} D_{28}^{-2} \epsilon_{B,-2}^{1 / 2} E_{53}^{5 / 4} n_{1,0}^{1 / 4} \Gamma_{\min , 2.5}^{-1} T_{\Delta, 2}^{-3 / 4} \mathrm{Jy},
\end{gathered}
$$

for the ISM case, and

$$
\begin{gathered}
\nu_{m} \sim 4.5 \times 10^{14}(1+z)^{-1 / 2}\left(\frac{p-2}{p-1}\right)^{2} \sqrt{\frac{(s+b-1)^{2}}{\alpha^{2} b(s-1)}} \epsilon_{e,-0.5}^{2} \epsilon_{B,-2}^{1 / 2} E_{53}^{-1 / 2} A_{*,-0.5} \Gamma_{\min , 2.5}^{2} T_{\Delta, 2}^{-1 / 2} \mathrm{~Hz}, \\
\nu_{c} \sim 4.0 \times 10^{14}(1+z)^{-3 / 2} \sqrt{\frac{\alpha^{6} b^{5}(s-1)}{(s+b-1)^{6}}} \epsilon_{B,-2}^{-3 / 2} E_{53}^{1 / 2} A_{*,-0.5}^{-2} T_{\Delta, 2}^{1 / 2} \mathrm{~Hz}, \\
F_{\nu, \max } \sim 6.5(1+z)^{2}\left[\frac{(s+b-1)(s-1)}{\alpha b s}\right] D_{28}^{-2} \epsilon_{B,-2}^{1 / 2} E_{53} A_{*,-0.5}^{1 / 2} \Gamma_{\min , 2.5}^{-1} T_{\Delta, 2}^{-1} \mathrm{Jy},
\end{gathered}
$$

for the wind case, where $T_{\Delta, 2}=T / 100 \mathrm{~s}$. The above expressions for the synchrotron radiation at the crossing time are quite similar to those in the UEM (e.g., Kobayashi 2000; Wu et al. 2003). We find that for a set of combinations of reasonable parameter values $(s=2.5,2,1.5$ and $b=2,1)$ the $t_{\mathrm{cm}}$ (the time when $\nu_{m}=\nu_{c}$ ) is always small for the ISM case, indicating that the reverse-shocked electrons are always in the slow cooling region. However, for the wind case, the electrons are usually fast cooling during the entire reverse shock phase, because $\nu_{m}$ is typically larger than $\nu_{c}$ at the crossing time and $\nu_{m}$ decreases with time 


\subsection{Thin Shell Case}

In the thin shell case, the reverse shock is always mildly-relativistic $\left(\gamma_{34}-1 \sim 1\right)$ and the crossing time depends on the crossing radius as $t_{\Delta} \propto r_{\Delta}^{(7+s-2 k) /(s+1)}$. For convenience, we choose $s=2$ to give the typical values of the two characteristic frequencies and peak flux density of synchrotron radiation,

$$
\begin{gathered}
\nu_{m} \sim 2.1 \times 10^{12}(1+z)^{-1}\left(\frac{p-2}{p-1}\right)^{2} \alpha^{4} \epsilon_{e,-0.5}^{2} \epsilon_{B,-2}^{1 / 2} \Gamma_{\min , 1.8}^{2} \mathrm{~Hz} \\
\nu_{c} \sim 7.1 \times 10^{16}(1+z)^{-1} \alpha^{-4 / 3} \epsilon_{B,-2}^{-3 / 2} E_{53}^{-2 / 3} n_{1,0}^{-5 / 6} \Gamma_{\min , 1.8}^{4 / 3} \mathrm{~Hz} \\
F_{\nu, \max } \sim 2.3(1+z) D_{28}^{-2} \epsilon_{B,-2}^{1 / 2} E_{53} n_{1,0}^{1 / 2} \Gamma_{\min , 1.8} \mathrm{Jy}
\end{gathered}
$$

for the ISM case, and

$$
\begin{gathered}
\nu_{m} \sim 4.2 \times 10^{12}(1+z)^{-1}\left(\frac{p-2}{p-1}\right)^{2} \alpha^{2} \epsilon_{e,-0.5}^{2} \epsilon_{B,-2}^{1 / 2} A_{*,-0.5}^{3 / 2} E_{53}^{-1} \Gamma_{\min , 1.8}^{4} \mathrm{~Hz} \\
\nu_{c} \sim 1.9 \times 10^{13}(1+z)^{-1} \alpha^{2} \epsilon_{B,-2}^{-3 / 2} E_{53} A_{*,-0.5}^{-5 / 2} \Gamma_{\min , 1.8}^{-2} \mathrm{~Hz} \\
F_{\nu, \max } \sim 385.0(1+z) D_{28}^{-2} \alpha^{-2} \epsilon_{B,-2}^{1 / 2} A_{*,-0.5}^{3 / 2} \Gamma_{\min , 1.8}^{3} \mathrm{Jy}
\end{gathered}
$$

for the wind case. It is shown in Table 1 that the temporal indices of $\nu_{m}, \nu_{c}$ and $F_{\max }$ are the same as in the varying injection model (Sari \& Mészáros 2000), which indicates that the $\Gamma$-distribution in the shell does not affect the shape of the light curve for the thin shell case (see Table 2) because the spreading effect erases the initial $\Gamma$-distribution.

\section{NUMERICAL RESULTS}

Since the thin shell case in our treatment has the same results as that in Sari \& Mészáros (2000), hereafter we just present the numerical results of the thick shell case. For the sake of simplicity, we only show the result by one set of parameters with $s=2$ and $b=2$, which is nevertheless sufficient to compare the result of the thick shell case in our paper to that of the thin shell case in the literature. We assume that the redshift of a GRB is $z=2$ since it is the average value of the observed GRBs in the Swift era (Le \& Dermer 2006). According to the standard shock acceleration mechanism, the energy index $p$ of electrons is about $2.2 \sim 2.3$, so we choose $p=2.3$.

We follow the method of Zou et al. (2005) to perform our numerical calculations with $\Gamma_{\max }=1000$, $\Gamma_{\min }=300, E_{0}=1.0 \times 10^{53} \mathrm{erg}, \Delta_{0}=1.0 \times 10^{13} \mathrm{~cm}, \epsilon_{e}=0.3, \epsilon_{B}=0.01, A_{*}=0.1, n_{1}=10 \mathrm{~cm}^{-3}$ and adopt the standard cosmology model with $\Omega_{m}=0.27, \Omega_{\Lambda}=0.73$ and $H_{0}=71 \mathrm{~km} \mathrm{~s}^{-1} \mathrm{Mpc}^{-1}$.

Figs. 2 and 3 show the light curves of synchrotron emission in the optical R band and X-ray band. To compare with the results of the UEM, we also plot the light curves of the UEM in the same energy band. In our calculations, the nonuniform and uniform ejecta have the same kinetic energy and total mass, so the uniform ejecta have a Lorentz factor of $s \Gamma_{\min } /(s-1)=600$. The upper panel denotes the R-band 
(1) In these two energy bands, there is a slow decay phase before the crossing time for the NUEM which is attributed to the energy injection by the low- $\Gamma$ part. (2) Before the crossing time, the reverse shock emission dominates the radiation in the optical band, while in the X-ray band, the shocks have comparable contribution. After that the forward shock emission gradually becomes important. This result is similar to the UEM. It is possible that the two shocked regions may have different microphysical parameters $\epsilon_{e}$ and $\epsilon_{B}$, which do not change the first conclusion but might change the second one.

Although we do not show the light curves of the thin shell case, we would like to emphasize the differences between the thick and thin shell cases. Since the reverse shock in the thin shell case is mildlyrelativistic all along and the flux density has the same behavior as presented by the refreshed shock scenario (Sari \& Mészáros 2000), a simple test of the thin shell case is that it predicts a maximal flux in the farinfrared or millimeter range a few hours to a few days after the GRB trigger. The forward shock emission contributes mainly in the high energy band and decays slower than that of the normal standard forward shock model. However, in the thick shell case, the reverse shock may be relativistic and dominate the flux in both the optical band and X-ray band at early times.

\section{DISCUSSION}

We have described the dynamics of radially structured ejecta interacting with the circum-burst medium through extending the method used in the UEM. It can be classified into two types: the thick shell case and the thin shell case, of which the latter is the same as in the UEM. Two parameters are introduced in the NUEM. One is the mass distribution index $s$ and the other is the Lorentz factor distribution index $b$. In our treatment, the thin shell case reproduces the same results obtained by Sari \& Mészáros (2000). On the other hand, in the thick shell case, the reverse shock could be relativistic or initially be non-relativistic and then become relativistic, contributing comparable radiation in the high energy band as the forward shock. Anyway, the energy injection induced by the nonuniform ejecta sweeping up the surrounding medium causes the light curves to decay more slowly.

Observationally, GRB990123 was seen to have a bright optical flash with initial flux decay as $F \propto t^{-2}$, which is attributed to the reverse shock emission, and subsequently the optical afterglow decays as $F \propto$ $t^{-1.1}$, which is mainly due to the forward shock emission. Both the thick and thin shell scenarios in the UEM can fit this optical flash well. The fast rise of $t^{3.4}$ can be explained if the circum-burst environment is homogenous ISM (Kobayashi 2000; Fan et al. 2002). However, some optical flashes (e.g., GRBs 021211, 050525a, 060111B and 060117B) have not been observed with this early rising part. This may be intrinsic, or due to late responses and slow slewing of optical telescopes. An early optical plateau was observed in a few GRB afterglows, e.g., GRBs 050319, 060206, 060210 and 060313, which may be attributed to the relativistic reverse shock emission of the radially structured ejecta sweeping up the circum-burst medium.

In Swift GRB afterglows, peculiar chromatic breaks have been universally observed, but the origin of these chromatic breaks is still an open question. Some models were proposed to explain the chromatic breaks, such as the model with evolving microphysical parameters, or the model in which the optical and 
involving reverse shock emission (Genet et al. 2007; Uhm \& Beloborodov 2007). Our current work shows that the chromatic breaks cannot be due to the reverse shock and we favor the former explanations.

Although the detailed prescription of a radially structured shell propagating into the circum-burst medium is presented in this paper, it should be noted that the shock-heated material separated by the contact discontinuity is assumed to be uniform. A more accurate solution of the reverse-forward shock interaction and emission needs a relativistic hydrodynamic simulation.

\section{ACKNOWLEDGMENTS}

This work was supported by the National Natural Science Foundation of China (grants 10473023, 10503012, 10621303, 10633040, and 10703002), the National Basic Research Program of China (973 Program 2009CB824800). XFW also thanks the support of the NSF AST 0307376, NASA NNX07AJ62G, NNX08AL40G, the China Postdoctoral Science Foundation, and the Postdoctoral Research Award of Jiangsu Province.

\section{References}

Akerlof C. et al., 1999, Nature, 398, 400

Blandford R. D., McKee, C. F., 1976, Phys. Fluids, 19, 1130

Chevalier R. A., Li Z. Y., 2000, ApJ, 536, 195

Costa E., et al., 1997, Nature, 387, 783

Fan Y. Z., Dai Z. G., Huang, Y. F., Lu, T., 2002, ChJAA, 2, 449

Genet F., Daigne F., Mochkovitch R., 2007, MNRAS, 381, 732

Jelinek M. et al., 2006, A\&A, 454, L119

Klotz A. et al., 2006, A\&A, 451, L39

Kobayashi S., Piran T., Sari R., 1999, ApJ, 513, 669

Kobayashi, S., 2000, ApJ, 545, 807

Le T., Dermer C. D., 2007, ApJ, 661, 394

Mészáros P., Rees M. J., 1993, ApJ, 405, 278

Mészáros P., Rees M. J., 1997, ApJ, 476, 232

Molinari E. et al., 2007, A\&A, 469, L13

Nousek J. A. et al., 2006, ApJ, 642,389

O’Brien P. T. et al., 2006, ApJ, 647, 1213

Panaitescu A., Mészáros P., Burrows, D. et al., 2006, MNRAS, 369, 2059

Racusin J. L. et al., 2008, Nature, 455, 183

Rees M. J., Mészáros P. 1992, MNRAS, 258, 41

Rees M. J.,Mészáros P., 1994, ApJ, 430, L93

Rees M. J.,Mészáros P., 1998, ApJ, 496, L1

Sari R., Piran T., 1999, ApJ,517, L109

Sari R.,Mészáros P., 2000, ApJ, 535, L33

Sari R., Piran T., 1995, ApJ, 455, L143

Shao L.,Dai Z. G., 2005, ApJ, 633, 1027

Uhm Z. L., Beloborodov A. M., 2007, ApJ, 665, L93

Wei D.M., 2003, A\&A, 400, 415

Wu X. F., Dai, Z. G., Huang, Y. F., Lu T., 2003, MNRAS, 342, 1131

Xue R. R., Fan Y. Z., Wei D. M., 2009, A\&A, 498, 671

Zhang B., Kobayashi, S., Mészáros P., 2003, ApJ, 595, 950

Zhang B., Fan Y, Z., Dyks J. et al., 2006, ApJ, 642, 354

Zhang B., 2007, ChJAA, 7, 1

Zou Y. C., Wu X. F., Dai Z. G., 2005, MNRAS, 363, 93 


\begin{tabular}{ccccc}
\hline \hline & \multicolumn{2}{c}{ Reverse Shock } & \multicolumn{2}{c}{ Forward Shock } \\
\hline & thick shell & thin shell & thick shell & thin shell \\
\hline$\nu_{m}$ & $\frac{5 k-b k-k s-16}{2 b(4-k)}$ & $-\frac{12-3 k+k s}{2(7-2 k+s)}$ & $\frac{(s-1-3 b)}{2 b}$ & $-\frac{24-7 k+k s}{2(7-2 k+s)}$ \\
\hline$\nu_{c}$ & $\frac{(3 k-4)(s+b-1)}{2 b(4-k)}$ & $\frac{(3 k-4)(s+1)}{2(7-2 k+s)}$ & $\frac{(3 k-4)(s+b-1)}{2 b(4-k)}$ & $\frac{(3 k-4)(s+1)}{2(7-2 k+s)}$ \\
\hline$F_{\nu, \max }$ & $\frac{k+b k-3 k s+10 s-6 b-2}{2 b(4-k)}$ & $\frac{3(k+2 s-4-k s)}{2(7-2 k+s)}$ & $\frac{3 k-b k-3 k s+8 s-8}{2 b(4-k)}$ & $\frac{k+6 s-3 k s-6}{2(7-2 k+s)}$ \\
\hline \hline
\end{tabular}

Table 1 Temporal indices of the peak frequency $\nu_{m}$, the cooling frequency $\nu_{c}$ and the peak flux density $F_{\nu, \max }$ for both the forward shock and reverse shock.

\begin{tabular}{lccc}
\hline \hline & & thick shell case \\
\hline & $\nu<\min \left[\nu_{\mathrm{m}}, \nu_{\mathrm{c}}\right]$ & $\min \left[\nu_{\mathrm{m}}, \nu_{\mathrm{c}}\right]<\nu<\max \left[\nu_{\mathrm{m}}, \nu_{\mathrm{c}}\right]$ & $\max \left[\nu_{\mathrm{m}}, \nu_{\mathrm{c}}\right]<\nu$ \\
\hline Slow Cooling & $\frac{5-9 b-k+2 b k+15 s-4 k s}{3 b(4-k)}$ & $\frac{12-12 b-3 k+3 b k-16 p+5 k p-b k p+20 s-5 k s-k p s}{4 b(4-k)}$ & $\frac{16-16 b-6 k+6 b k-16 p+5 k p-b k p+16 s-2 k s-k p s}{4 b(4-k)}$ \\
Fast Cooling & $\frac{-5-7 b+3 k+17 s-6 k s}{3 b(4-k)}$ & $\frac{-16 b-k+5 b k+16 s-3 k s}{4 b(4-k)}$ & $\frac{16-16 b-6 k+6 b k-16 p+5 k p-b k p+16 s-2 k s-k p s}{4 b(4-k)}$ \\
\hline \hline Slow Cooling & $\frac{12-3 k-9 s+4 k s}{6 k-3(7+s)}$ & $\frac{12-3 k+12 p-3 k p-12 s+5 k s+k p s}{8 k-4(7+s)}$ & $\frac{16-6 k+12 p-3 k p-8 s+2 k s+k p s}{8 k-4(7+s)}$ \\
Fast Cooling & $\frac{16-3 k-11 s+6 k s}{6 k-3(7+s)}$ & $\frac{28-9 k-8 s+3 k s}{8 k-4(7+s)}$ & $\frac{16-6 k+12 p-3 k p-8 s+2 k s+k p s}{8 k-4(7+s)}$ \\
\hline \hline
\end{tabular}

Table 2 Temporal indices of the flux density $F_{\nu} \propto t^{\alpha} \nu^{\beta}$ of synchrotron radiation from a reverse shock. Both the thick shell case and the thin shell case are considered.

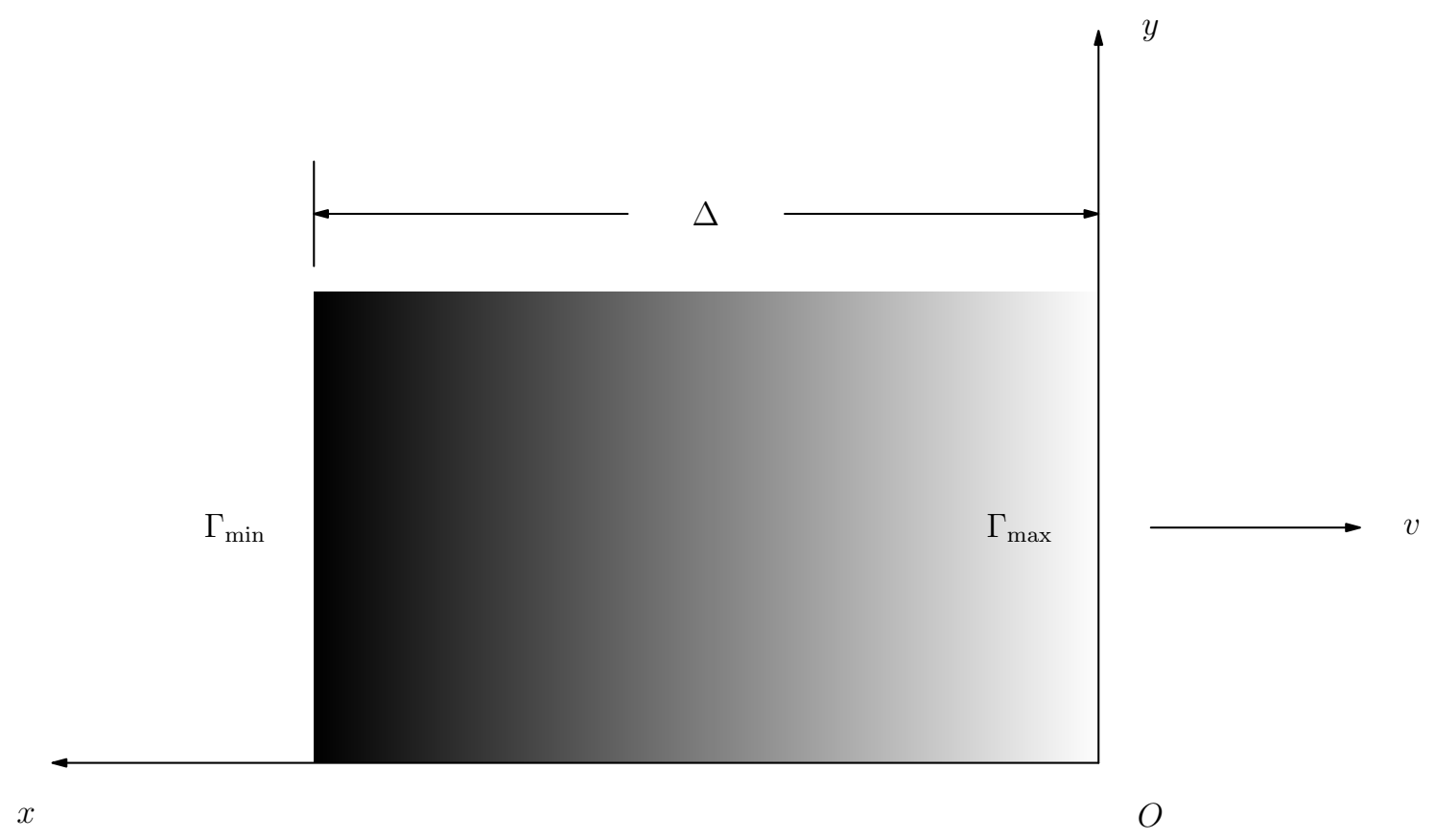

Fig. 1 Schematic of the radially structured shell. $\Delta$ is the width of the shell, $\Gamma_{\min }$ and $\Gamma_{\max }$ are the Lorentz factors on the edge of the shell respectively. The part with the lower Lorentz factor in the shell has more kinetic energy (marked with darker gray) than that with the higher Lorentz factor. 


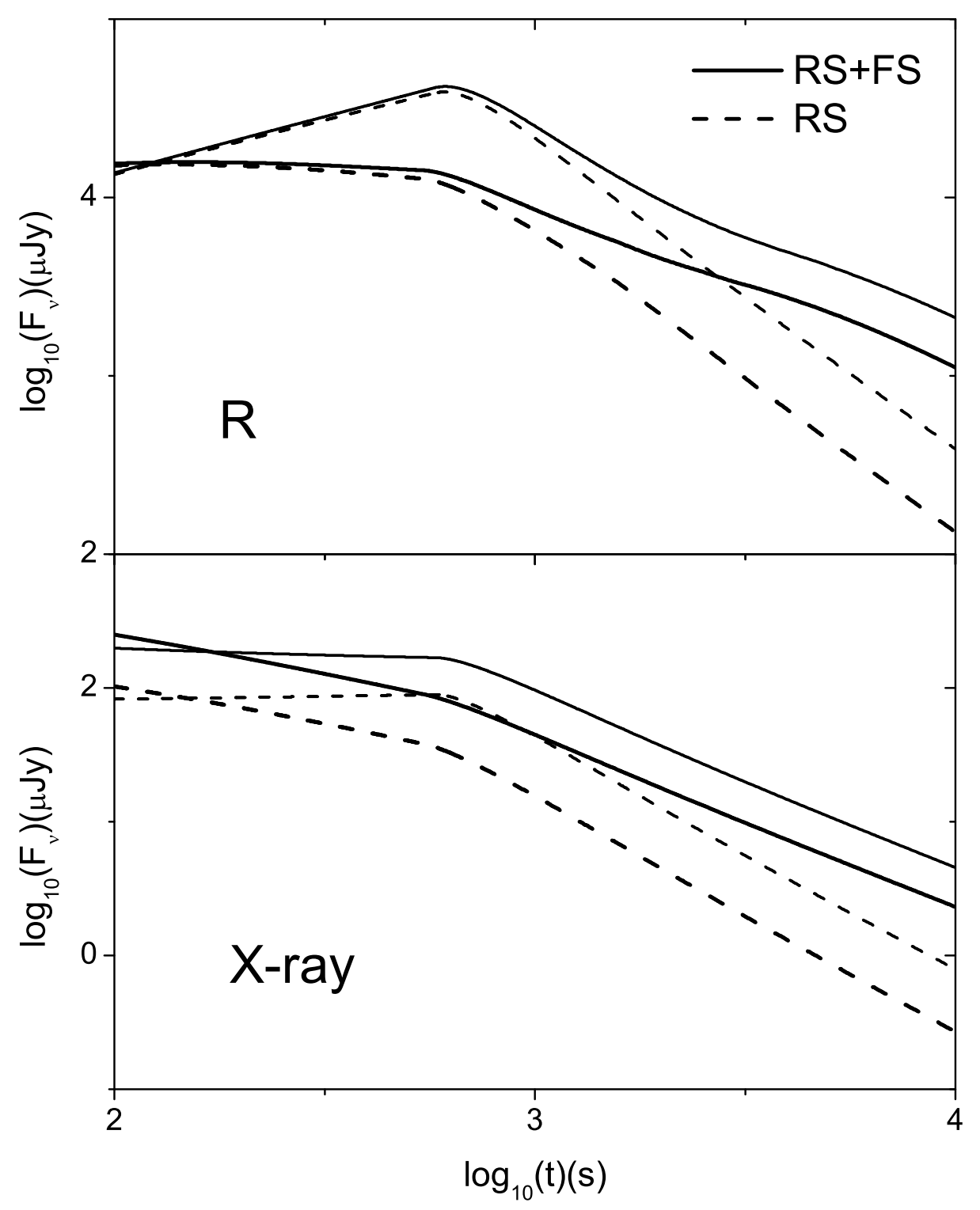

Fig. 2 Synchrotron radiation flux density in the R band and X-ray band as a function of time for the ISM case. Solid lines represent the whole emission from both the forward shock and the reverse shock. Dashed lines represent the contribution from the reverse shock. Thick lines are for the NUEM while thin lines are for the UEM. 


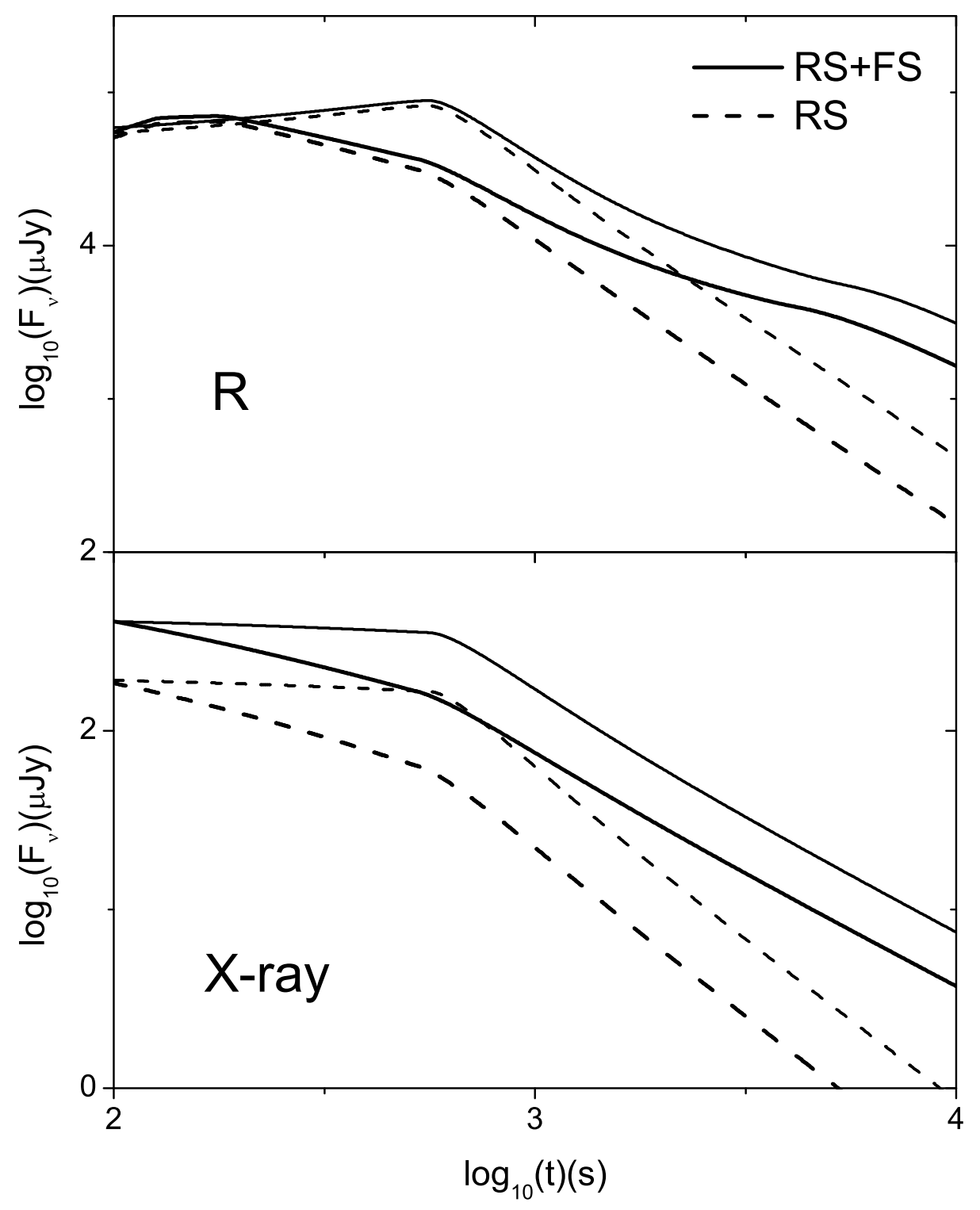

Fig. 3 Synchrotron radiation flux density in the $\mathrm{R}$ band and $\mathrm{X}$-ray band as a function of time, the same as in Fig. 2. for the wind case. Parameters are given in the text. 